\title{
Sonoterapia en la reducción de la ansiedad y el dolor posoperatorio en pacientes con anestesia regional como técnica única: ensayo clínico aleatorizado y controlado
}

\author{
Sonotherapy in the reduction of anxiety and postoperative pain in patients with regional \\ anesthesia as a sole technique: randomized, controlled clinical trial
}

\author{
Ana S. Arango-Gutiérrez', Laura J. Buitrago-Cifuentes' ${ }^{1}$, Ana M. Medina-Hinestroza ${ }^{1}$, \\ Sergio A. Molina-Paniagua1, Eliseo Moreno², Johan S. Rivera-Díaz ${ }^{3}$, Elsa M. Vásquez-Trespalacios ${ }^{*}$, \\ Mario A. Arcila-Lotero ${ }^{3}$ y María P. González-Obregón ${ }^{3}$ \\ ${ }^{1}$ Programa de Anestesiología, Universidad CES; ${ }^{2}$ Facultad de Medicina, Universidad CES; ${ }^{3}$ Servicio de Anestesiología, Instituto Colombiano del \\ Dolor, Universidad CES; ${ }^{4}$ Magíster en Epidemiología, Departamento de investigación, Universidad CES. Medellín, Colombia
}

\begin{abstract}
Resumen
Objetivo: Evaluar la eficacia de la sonoterapia en la diminución de la ansiedad y el dolor posoperatorio en adultos llevados a cirugía ambulatoria de ortopedia bajo anestesia regional. Método: estudio aleatorizado, doble ciego, controlado con placebo, en 80 pacientes adultos sometidos a cirugía ortopédica; 40 recibieron intervención con sonoterapia y 40 no la recibieron. Se midieron el dolor y la ansiedad en el preoperatorio, en el posoperatorio inmediato, al alta, y 24 y 48 horas después. Se midieron parámetros hemodinámicos en cuatro momentos. Resultados: No se encontraron diferencias significativas entre ambos grupos en las escalas de dolor ni ansiedad. Se observó una reducción significativa de la presión arterial sistólica luego de la aplicación de la intervención y en recuperación. No se observaron diferencias en otras variables hemodinámicas entre los grupos, pero la reducción de algunas ocurre de forma más rápida en el grupo de intervención. Conclusiones: En pacientes adultos llevados a cirugía ambulatoria de ortopedia bajo anestesia regional, la sonoterapia es una estrategia novedosa que reduce la presión arterial sistólica, y esto puede considerarse como un indicador de disminución de la ansiedad. En nuestro estudio no se pudo demostrar diferencia en términos de control del dolor ni de requerimiento de sedación o analgesia adicional.
\end{abstract}

PALABRAS CLAVE: Musicoterapia. Bloqueo nervioso. Dolor. Ansiedad. Atención ambulatoria. Ensayo clínico. Adulto.

\begin{abstract}
Objective: To determine if sonotherapy reduces the anxiety level and postoperative pain in adults undergoing outpatient orthopedic surgery under regional anesthesia. Method: A randomized, double-blind, placebo-controlled study in 80 adult patients undergoing orthopedic surgery, 40 received intervention with sonotherapy and 40 did not receive it. Pain, anxiety in the preoperative period, immediate postoperative, high, 24 and 48 hours later were measured. Hemodynamic parameters were measured in four moments. Results: No significant differences were found between the two groups in the pain scales neither anxiety. A significant reduction of the systolic blood pressure was observed after the application of sonotherapy and in recovery. There was no difference in other hemodynamic variables such as heart rate, respiratory rate, arterial oxygen saturation or diastolic blood pressure between groups, however, the observed changes in some of them occurred faster in the intervention
\end{abstract}

\footnotetext{
Correspondencia:

*Elsa M. Vásquez-Trespalacios

Calle 10르 \#22-04

Medellín, Colombia

E-mail: evasquez@ces.edu.co
}

Fecha de recepción: 10-09-2018

Fecha de aceptación: 06-05-2019

DOI: $10.24875 / C I R U .19000715$
Cir Cir. 2019;87:545-553

Contents available at PubMed www.cirugiaycirujanos.com 
group. Conclusions: In adult patients undergoing outpatient orthopaedic surgery under regional anaesthesia, sound therapy is a novel strategy that significantly reduces systolic blood pressure, considered as an indicator of decreased anxiety. However, in our study, no difference could be demonstrated in terms of pain control or the need for additional sedation or analgesia.

KEY WORDS: Music therapy. Nerve block. Pain. Anxiety. Ambulatory care. Clinical trial. Adult.

\section{Introducción}

El dolor posoperatorio agudo es una respuesta normal a una intervención quirúrgica, y es una causa de retraso en la recuperación y en el alta después de la cirugía; también aumenta el riesgo de infección de las heridas y de complicaciones respiratorias y cardiovasculares ${ }^{1,2}$.

La incidencia de dolor posoperatorio agudo varía ampliamente entre individuos y procedimientos, conociéndose que una gran proporción de pacientes posquirúrgicos sufren dolor agudo moderado a intenso ${ }^{3}$, y entre un 20 y un $56 \%$ pueden desarrollar un dolor crónico luego de la cirugía 4 .

El dolor posoperatorio intenso puede llegar a estar presente en aproximadamente el $70 \%$ de los pacientes en algún momento de su recuperación, y en el 30\% un dolor moderado luego de ser sometidos a un procedimiento quirúrgico. Un $58 \%$ de los pacientes presentan dolor moderado o intenso en el momento de abandonar la sala de recuperación posanestésica ${ }^{5}$.

En cirugía ortopédica, las técnicas anestésicas regionales con bloqueo de nervio periférico han venido en aumento dadas sus ventajas sobre la anestesia general, presentando mejor control del dolor posoperatorio, disminución en el consumo de opiáceos, menor incidencia de náuseas y vómitos posoperatorios $^{6}$, y menor tiempo de estancia en la sala de recuperación posanestésica; asimismo, la preferencia y la satisfacción de los pacientes se inclina hacia las técnicas de anestesia regional ${ }^{7}$.

Sumado a lo anterior, la ansiedad perioperatoria trae consigo estrés fisiológico y disminuye el umbral del dolor, por lo que los pacientes requerirán mayores dosis de analgésicos y esto podría resultar en un aumento de las complicaciones posoperatorias ${ }^{8,9}$.

Se han evaluado diferentes técnicas farmacológicas y no farmacológicas para el manejo del dolor posoperatorio y la ansiedad perioperatoria. En las últimas décadas se han estudiado la música y las ondas sonoras como posible estrategia para disminuir el dolor posquirúrgico. La sonoterapia o terapia con ondas sonoras es una opción para el cuidado del paciente, para la mejoría de síntomas como la ansiedad y el dolor, y para el aumento de la calidad de vida ${ }^{10-14}$.
El objetivo de este estudio es evaluar el efecto de la sonoterapia sobre el nivel de ansiedad perioperatoria, el dolor posoperatorio, los parámetros hemodinámicos y el requerimiento de opiáceos en los pacientes llevados a cirugía ortopédica ambulatoria bajo anestesia regional en el Instituto Colombiano del Dolor en Medellín, Colombia.

\section{Método}

Después de elaborar el consentimiento informado y obtener la aprobación del Comité de Ética de la Universidad CES (Noviembre 15, 2016, código 645), se estudiaron 80 pacientes programados para cirugía electiva ambulatoria de ortopedia bajo anestesia regional en el Instituto Colombiano del Dolor a través de un ensayo clínico aleatorizado, de quienes se obtuvo consentimiento informado verbal y escrito. El protocolo está registrado en ClinicalTrials.gov NCT03512587. Los procedimientos fueron realizados en concordancia con la Declaración de Helsinki.

Se incluyeron pacientes de 18 a 60 años, con un riesgo anestésico según la clasificación de la Sociedad Americana de Anestesia 1 y 2 , sin contraindicaciones para la administración de opiáceos ni benzodiacepinas. Además, los pacientes debían vivir en el área metropolitana con acceso a líneas telefónicas para la realización de los controles a las 24 y 48 horas. También fue necesario que presentaran un nivel de compresión adecuado para los controles telefónicos y un entendimiento de las escalas numéricas. Los criterios de exclusión fueron la presencia de problemas auditivos o cognitivos, el requerimiento de anestesia general en algún momento del intraoperatorio, la obesidad mórbida (índice de masa corporal > 40) y el uso de ansiolíticos en el último mes.

Se realizó una aleatorización simple con una tabla de números generada con el software Epidat (4.2). La asignación de los grupos aleatorizados se guardó dentro de sobres oscuros por parte de un integrante del equipo de investigación. Uno de los grupos recibió la intervención con sonoterapia, que se reprodujo a través de un MP3 desde antes de la realización de la anestesia regional hasta el alta. El otro grupo no 


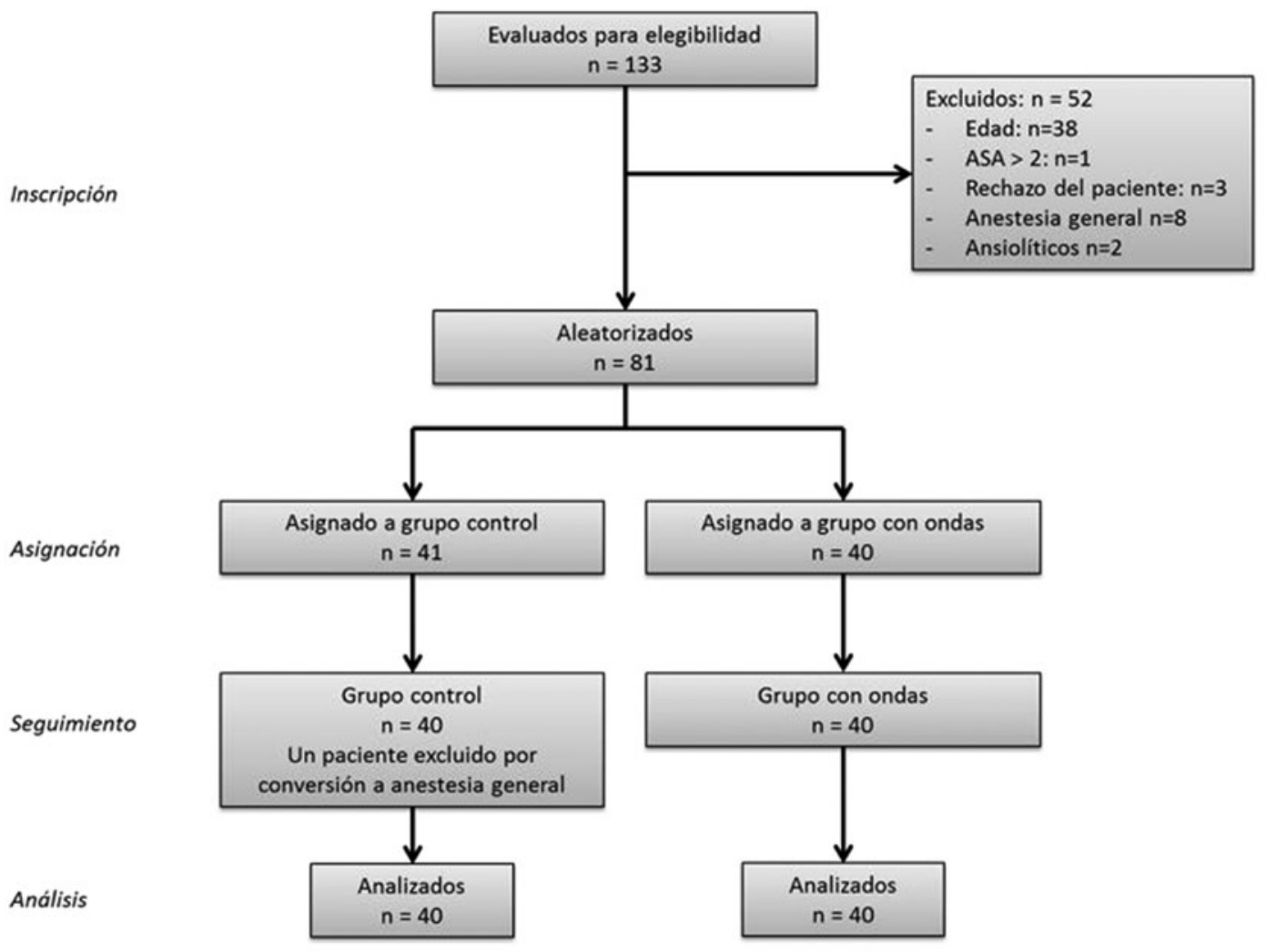

Figura 1. Algoritmo del estudio de acuerdo con el flujograma CONSORT.

recibió las ondas sonoras, pero con el fin de mantener el cegamiento de los pacientes, el anestesiólogo y el grupo quirúrgico, se les colocaron igualmente los audífonos con el MP3, el cual no reproducía ningún sonido (Fig. 1).

Se midieron variables hemodinámicas, como frecuencia cardiaca, frecuencia respiratoria, presión arterial y saturación arterial de oxígeno en cuatro momentos: antes de la aplicación de las ondas (momento 1), después de la aplicación de las ondas (momento 2), al ingreso a recuperación después de la cirugía (momento 3) y antes del alta (momento 4).

Igualmente se realizó la evaluación del dolor con una escala visual análoga $(E V A)^{9}$ y de la ansiedad perioperatoria por medio del cuestionario para estado ansioso STAI (escala de Spielberger) ${ }^{10}$. La escala STAI clasifica el grado de ansiedad en estado ansioso y rasgo ansioso; para el estudio utilizamos la medición del estado ansioso de acuerdo con un cuestionario de 20 preguntas. Los resultados permiten clasificar el estado ansioso en leve, moderado o grave según el puntaje total.

El dolor y la ansiedad se midieron, a través de las escalas previamente descritas, en cuatro momentos: antes de la aplicación de las ondas (momento 1), antes del alta (momento 2), a las 24 horas (momento 3) y a las 48 horas del posoperatorio (momento 4). La evaluación después del alta se hacía mediante llamadas telefónicas en las que se realizaba nuevamente el cuestionario y se evaluaba el dolor. Las llamadas telefónicas fueron efectuadas por investigadores que desconocían el grupo al que estaba asignado cada paciente.

Los análisis estadísticos se realizaron con el programa SPSS versión 21 (SPSS Inc., Chigaco, IL, EE.UU.). Las variables continuas se analizaron mediante la prueba $t$ de student 0 la prueba $U$ de Mann-Whitney, dependiendo de la distribución de la variable. Las variables categóricas se analizaron con la prueba de ji al cuadrado o con la prueba exacta de Fisher, cuando fuera apropiado, para la comparación entre grupos. Se aplicó la prueba Anova de dos factores (tiempo y tratamiento). Las comparaciones intragrupo e intergrupos se realizaron mediante pruebas de contrastes posteriores ajustadas por comparaciones múltiples para la evaluación del dolor y la ansiedad en los cuatro momentos considerados, según lo descrito por Leys y Schumann ${ }^{15}$. Se consideró estadísticamente significativo un valor de $p<0.05$. Se realizó un análisis por intención de tratar. 


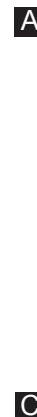

C
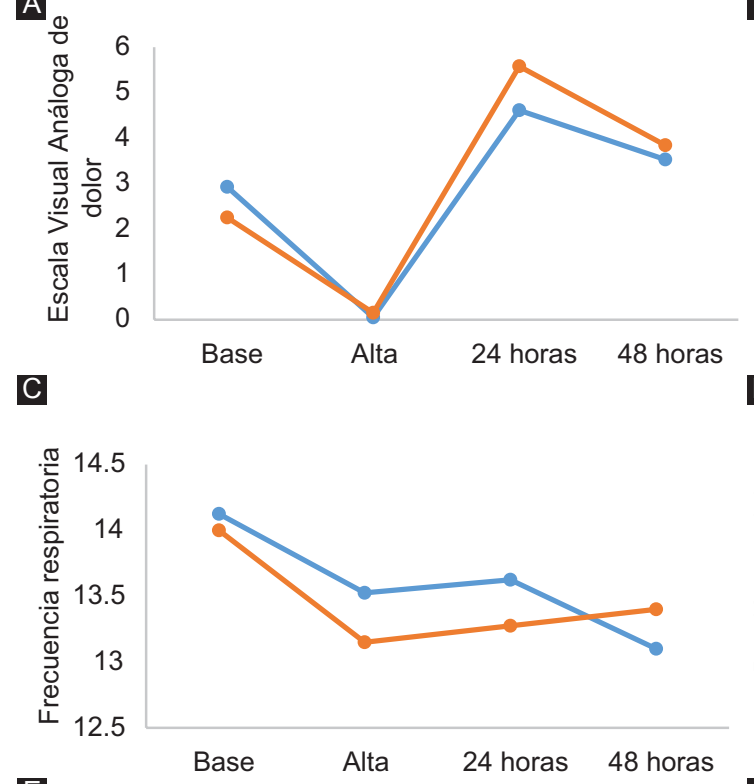

E

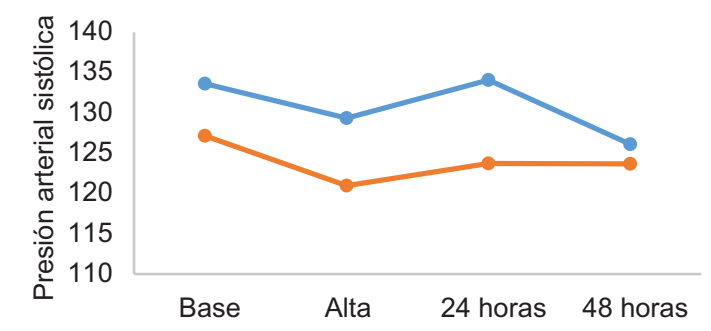

B

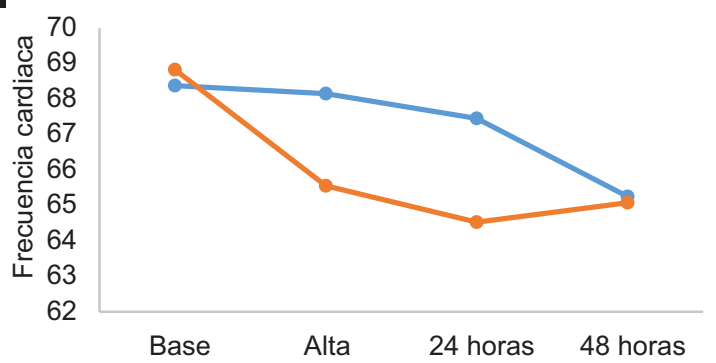

D

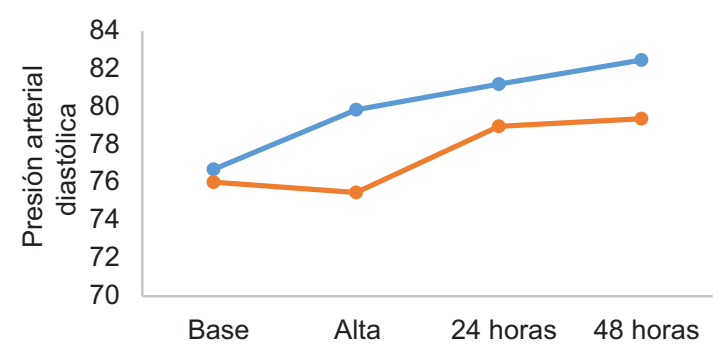

$\mathrm{F}$

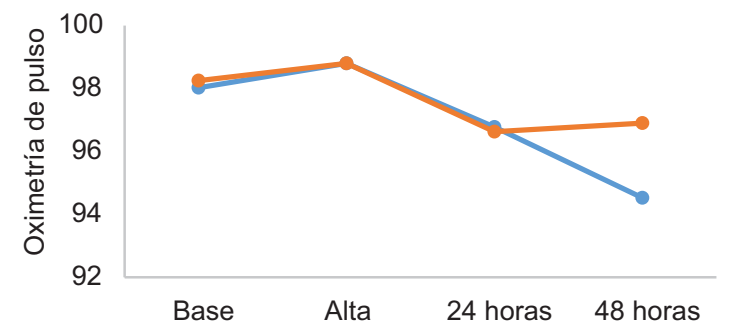

G

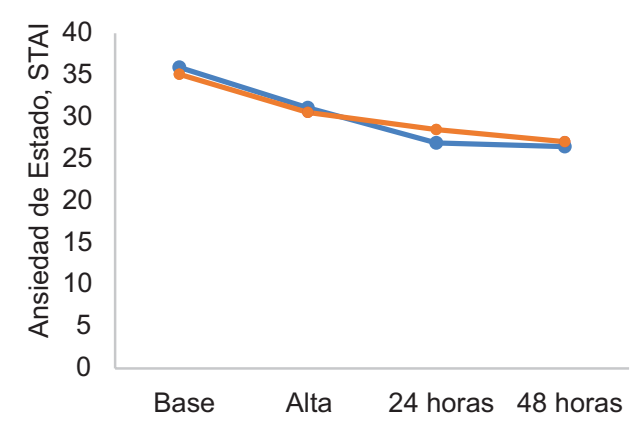

Figura 2. Cambio en los parámetros medidos en los cuatro momentos. Línea azul: grupo control. Línea naranja: grupo sonoterapia. A: Dolor en la escala visual análoga. B: Frecuencia cardíaca. C: Frecuencia respiratoria. D: Presión arterial diastólica. E: Presión arterial sistólica. F: Oximetría de pulso. G: Ansiedad de estado STAI (Cuestionario de Ansiedad Estado Rasgo).

\section{Resultados}

Se evaluaron 133 pacientes llevados a cirugía electiva ambulatoria de ortopedia bajo anestesia regional entre febrero y marzo de 2017, de los cuales 52 no participaron por presentar uno o más criterios de exclusión. Los 81 pacientes restantes se aleatorizaron en dos grupos: 41 para el grupo control y 40 para recibir la sonoterapia cuántica personalizada. De los pacientes asignados al grupo control, uno se excluyó por conversión a anestesia general (Fig. 1). Los dos grupos fueron comparables en términos de edad, sexo, antecedente de dolor crónico y uso de opiáceos en los últimos 3 meses, sin presentar diferencias estadísticamente significativas (Tabla 1).

Los procedimientos más frecuentes fueron cirugías de mano (48; $60 \%)$, hombro (14; $17.5 \%)$, pie $(7 ; 8.75 \%)$ y antebrazo (6; 7.5\%). Los bloqueos más frecuentes, tanto para el grupo general como para los subgrupos, fueron axilar, interescalénico y supraclavicular. No se encontró diferencia estadísticamente significativa en 
Tabla 1. Características de los participantes

\begin{tabular}{|c|c|c|c|}
\hline & Grupo control $(n=40)$ & Grupo sonoterapia $(n=40)$ & $\mathbf{p}$ \\
\hline Edad, años (promedio \pm DE) & $42.05 \pm 13.3$ & $42.45 \pm 11.10$ & 0.88 \\
\hline \multicolumn{4}{|l|}{ Sexo, n (\%) } \\
\hline - Femenino & $18(45)$ & $18(45)$ & 1.00 \\
\hline - Masculino & $22(55)$ & $22(55)$ & \\
\hline \multicolumn{4}{|l|}{ Bloqueos, n (\%) } \\
\hline - Axilar & $23(57.5)$ & $24(60.0)$ & 0.70 \\
\hline - Interescalénico & $7(17.5)$ & $7(17.5)$ & \\
\hline - Supraclavicular & $3(7.5)$ & $5(12.5)$ & \\
\hline - Ciático poplíteo + safeno & $3(7.5)$ & $4(10)$ & \\
\hline - Ciático poplíteo + femoral & $1(2.5)$ & $0(0)$ & \\
\hline - Ciático subglúteo + femoral & $1(2.5)$ & $0(0)$ & \\
\hline - Ciático subglúteo & $1(2.5)$ & $0(0)$ & \\
\hline - Ciático subglúteo + femoral + obturador + femorocutáneo & $1(2.5)$ & $0(0)$ & \\
\hline Dolor crónico, n (\%) & $9(22.5)$ & $6(15)$ & 0.64 \\
\hline Tipo de cirugía, n (\%) & & & 0.52 \\
\hline - Hombro & $7(17.5)$ & $7(17.5)$ & \\
\hline - Codo & $1(2.5)$ & $0(0)$ & \\
\hline - Antebrazo & $3(7.5)$ & $3(7.5)$ & \\
\hline- Mano & $22(55)$ & $26(65)$ & \\
\hline - Rodilla & $2(5)$ & $0(0)$ & \\
\hline - Pierna & $1(2.5)$ & $0(0)$ & \\
\hline - Tobillo & $1(2.5)$ & $0(0)$ & \\
\hline - Pie & $3(7.5)$ & $4(10)$ & \\
\hline Duración de la cirugía, min (promedio \pm DE) & $67.88 \pm 40.06$ & $56.33 \pm 29.50$ & 0.14 \\
\hline Sedación adicional, n (\%) & $7(17.5)$ & $11(27.5)$ & 0.28 \\
\hline Tiempo en recuperación, min (promedio \pm DE) & $31.68 \pm 14.97$ & $32.60 \pm 14.16$ & 0.77 \\
\hline Opiáceos 3 meses, n (\%) & $4(10)$ & $1(2.5)$ & 0.16 \\
\hline
\end{tabular}

cuanto al tipo de cirugía ni de bloqueo utilizado para el procedimiento.

\section{Comparación entre los grupos de estudio}

\section{DisminuCIÓN DEL DOLOR}

Los pacientes que recibieron la sonoterapia no mostraron una disminución en la EVA estadísticamente significativa en comparación con el grupo control antes del alta $(p=0.60)$, a las 24 horas $(p=0.57)$ ni a las 48 horas posoperatorias $(p=0.70)$. La reducción del dolor a las 48 horas no se esperaba, dada la duración de acción del bloqueo y de la intervención (Fig. 2).

\section{REDUCCIÓN DE LA ANSIEDAD}

No se encontró diferencia significativa en los estados de ansiedad antes de la intervención $(p=0.78)$, antes del alta $(p=0.51)$ ni a las 48 horas posoperatorias $(p=0.60)$, pero sí a las 24 horas a favor del grupo que recibió placebo $(p=0.046)$.

\section{Parámetros hemodinámicos}

En la evaluación de los diferentes momentos hemodinámicos se encontró una reducción significativa de la presión arterial sistólica tras la aplicación de las ondas sonoras $(p=0.021)$ y en recuperación $(p=0.006)$ (Fig. 2), sin encontrar diferencia de la presión arterial diastólica, la frecuencia cardiaca, la frecuencia respiratoria ni la saturación periférica de oxígeno.

\section{NECESIDAD DE OPIÁCEOS}

Solo un paciente del grupo de intervención requirió opiáceos de rescate en el perioperatorio, sin ser 
estadísticamente significativo ( $p=0.31)$. No se encontró diferencia en el requerimiento de sedación adicional, la duración del procedimiento quirúrgico ni el tiempo de recuperación. En ninguno de los grupos de estudio se presentaron complicaciones.

Cuando se realiza el análisis de los parámetros analizados en los cuatro momentos de tiempo, puede observarse que tanto la frecuencia cardiaca como la respiratoria y la presión arterial sistólica y diastólica presentan una disminución más temprana en los participantes del grupo intervenido con sonoterapia que en el grupo control (Fig. 2).

\section{Pruebas de medidas Repetidas}

Con respecto al cambio en las mediciones de los parámetros en los cuatro momentos de tiempo, se observa que el dolor al alta, a las 24 horas y a las 48 horas en ambos grupos cambia de forma estadísticamente significativa con respecto al dolor reportado en la línea de base.

En el grupo que recibió la intervención con sonoterapia se observa una disminución estadísticamente significativa al alta y a las 24 horas con respecto a la línea basal $(p<0.05)$. La presión arterial sistólica presenta la misma tendencia en el grupo de sonoterapia y es significativamente diferente del promedio para el grupo control.

El estado ansioso, medido por la escala STAI, cambia de forma estadísticamente significativa con respecto al estado basal en ambos grupos (Tabla 2).

\section{Discusión}

El dolor es una experiencia sensorial subjetiva con múltiples elementos que pueden modificar su percepción, su modulación y la reacción ante él, tales como los afectos, el temperamento, el carácter y la exposición a situaciones dolorosas ${ }^{16}$.

También, en el periodo perioperatorio, el paciente experimenta ansiedad como una respuesta fisiológica al enfrentarse a un ambiente desconocido y ante la posibilidad de desenlaces inciertos respecto a su salud y proceso de enfermedad. Este estrés genera impulsos en el hipotálamo que activan el eje hipotalámico-hipofisiario-adrenal, y aumenta la producción de cortisol y de otros mediadores humorales. Cuando no hay una adecuada regulación de la respuesta a la ansiedad puede alterarse la cicatrización y aumentar los requerimientos anestésicos y los riesgos derivados de la anestesia. A su vez, pueden necesitarse dosis mayores de analgésicos o adoptar posturas antiálgicas que se derivan en menor tiempo de deambulación y aumento en el tiempo de recuperación y en los días de estancia hospitalaria o de incapacidad ${ }^{8}$.

Con la intención de lograr un abordaje integral de los pacientes se han utilizado técnicas no farmacológicas que permiten realizar un manejo analgésico y ansiolítico al mismo tiempo. En las últimas décadas se han estudiado la música y las ondas sonoras como posible estrategia para disminuir el dolor posquirúrgico. Precisamente, la musicoterapia o sonoterapia es una opción para el cuidado del paciente que se ha empleado para disminuir el dolor desde varios años atrás. Diversos hospitales, unidades de cuidados intensivos, departamentos de psiquiatría y oncología, entre otros, utilizan esta estrategia y se recomienda en diferentes ámbi$\operatorname{tos}^{17,18}$. La mayoría de las recomendaciones sugieren música relajante o sonidos de la naturaleza, puesto que al carecer de letra evitan la evocación de sucesos o recuerdos que alteren la percepción de los pacientes ${ }^{13,14,19}$.

El objetivo de este estudio era evaluar sí existían diferencias en el dolor, la ansiedad, el requerimiento de opiáceos y los parámetros hemodinámicos entre un grupo intervenido con sonoterapia y un grupo control.

Como hallazgos principales, no se encontraron diferencias estadísticamente significativas en el dolor medido a través de la EVA y la ansiedad medida por la escala STAI entre los dos grupos de tratamiento, durante los cuatro momentos de tiempo, de modo similar a lo reportado por otros estudios que no refieren diferencias significativas en el estado de ansiedad cuando aplican la intervención a pacientes programados para cirugía ortopédica, oncológica o en unidades de cuidados intensivos, comparados con un grupo sin intervención ${ }^{20-22}$ o con otras intervenciones diferentes de la música ${ }^{23,24}$. En cambio, otros estudios encuentran una disminución estadísticamente significativa en la escala de dolor y no reportan diferencias en el grado de ansiedad ${ }^{25,26}$.

Un metaanálisis que incluyó 26 estudios que comparaban la intervención con música señala que esta puede tener un efecto benéfico en el estado de ansiedad preoperatoria, logrando en conjunto una reducción 5.72 unidades mayor que en el grupo control cuando se utilizó la escala STAI y de -0.60 unidades estandarizadas cuando se utilizaron otras 
Tabla 2. Comparación del dolor, las características hemodinámicas y la ansiedad

\begin{tabular}{llcccc}
\hline & Grupo & Línea base Media (DE) & Alta Media (DE) & 24 horas Media (DE) & 48 horas Media (DE) \\
\hline Dolor, escala visual análoga & Control & $2.9(3.3)$ & $0.1(0.3)^{\mathrm{a}}$ & $4.6(3.1)^{\mathrm{a}}$ & $3.5(3)^{\mathrm{a}}$ \\
& Sonoterapia & $2.3(3.2)$ & $0.2(0.7)^{\mathrm{a}}$ & $5.6(2.9)^{\mathrm{a}}$ & $3.8(2.6)^{\mathrm{a}}$ \\
Frecuencia cardiaca & Control & $68.4(9.6)$ & $68.2(10.8)$ & $67.5(10.5)$ & $65.3(14.7)$ \\
& Sonoterapia & $68.8(8.5)$ & $65.6(8.6)$ & $64.5(8.3)$ & $65.1(8.2)$ \\
Frecuencia respiratoria & Control & $14.1(1)$ & $13.5(1.4)$ & $13.6(1.2)$ & $13.1(2.3)^{\mathrm{a}}$ \\
& Sonoterapia & $14(1.2)$ & $13.2(1.6)^{\mathrm{a}}$ & $13.3(1.2)^{\mathrm{a}}$ & $13.4(1.2)$ \\
Presión arterial diastólica & Control & $19(10.3)$ & $21.9(10.7)$ & $26.9(11.3)$ & $25.8(22.4)^{\mathrm{a}}$ \\
& Sonoterapia & $18.7(10.9)$ & $21.4(11)$ & $25.6(11.1)$ & $25.5(10.7)$ \\
Presión arterial sistólica & Control & $133.6(15.2)$ & $129.4(17.3)$ & $134.1(18)$ & $126.1(26.4)$ \\
& Sonoterapia & $127.2(14.6)$ & $121(14.3)^{\mathrm{b}}$ & $123.7(14.4)^{\mathrm{b}}$ & $123.7(16.4)$ \\
Oximetría de pulso & Control & $98(1.2)$ & $98.8(1.1)$ & $96.8(1.8)$ & $94.5(15.4)^{\mathrm{a}}$
\end{tabular}

DE: desviación estándar.

escalas, de forma contraria a lo observado en este estudio $^{27}$. Este mismo metaanálisis también reporta un efecto pequeño de la intervención con música sobre parámetros como la presión arterial diastólica y la frecuencia cardiaca, pero no en la presión arterial sistólica, la frecuencia respiratoria y la temperatura corporal.

El mayor efecto en la reducción del dolor y la ansiedad perioperatoria se logra cuando los pacientes son quienes eligen la música que desean escuchar de una lista que se les suministra ${ }^{18,28}$. En nuestro estudio, las ondas sonoras que escuchaban los pacientes estaban predeterminadas.

Es importante señalar que en nuestro estudio se encontraron diferencias estadísticamente significativas en la presión arterial sistólica entre los grupos, siendo menor en el que recibió sonoterapia, de modo similar a lo referido en estudios semejantes ${ }^{5,29-31}$. Esta disminución en la presión arterial sistólica puede reflejar una baja en la excitación simpática correspondiente a un estado de disminución de la ansiedad.

Otro metaanálisis realizado específicamente con estudios que buscaban la reducción de la ansiedad y del dolor en pacientes sometidos a biopsia reporta que la intervención con música tiene una tendencia hacia la diminución de la presión arterial sistólica antes de la biopsia, y sobre la ansiedad, la presión arterial sistólica y la frecuencia cardiaca posteriores a la biopsia. Igualmente, la música es efectiva para la reducción del dolor luego de la biopsia, con una calidad de la evidencia evaluada como moderada ${ }^{32}$.

Entre los grupos no se encontraron diferencias significativas en el requerimiento de opiáceos ni en el tiempo de recuperación, de modo similar a lo encontrado por Gallagher, et al. ${ }^{33}$.

La multiplicidad de cirugías llevadas a cabo en los pacientes de este estudio puede ser un factor que explique la ausencia de diferencias estadísticamente significativas en el dolor y la ansiedad entre los grupos; es probable que en estudios más controlados al respecto la diferencia pueda ser más claramente verificable.

Como perspectivas futuras es importante explorar en profundidad los cambios fisiológicos que se producen en los pacientes programados para cirugía ortopédica como indicadores de reducción en los estados de ansiedad, así como la utilización de instrumentos que permitan medir estos estados y que sean altamente sensibles a cambios en tiempos cortos de medición, ya que los mecanismos fisiológicos por los cuales la música logra una reducción de la frecuencia cardiaca no están completamente dilucidados ${ }^{34}$.

Existen algunas limitaciones, tales como la recolección y la tabulación de los datos; sin embargo, se tomaron las medidas respectivas para disminuir su 
presentación, puesto que los instrumentos de recolección de la información se estandarizaron desde el principio. Se controló el sesgo de información de los pacientes explicándoles la posibilidad de escuchar o no las ondas para impedir que comunicaran al anestesiólogo y al grupo quirúrgico en qué grupo quedaron aleatorizados. También se realizó la toma de las variables hemodinámicas con los mismos equipos de monitoreo, previamente calibrados por la institución. Al tener multiplicidad de variables, su análisis puede generar confusión. Sin embargo, se tomaron las medidas respectivas para guiar dicho análisis.

El efecto placebo en este tipo de estudios no puede ser descartado, dado que los desenlaces como dolor y ansiedad son recolectados por autorreporte de los pacientes.

\section{Conclusiones}

Este ensayo clínico aleatorizado y doble ciego muestra que, en los pacientes que son llevados a cirugía ortopédica ambulatoria bajo anestesia regional como técnica única, la sonoterapia es una estrategia que reduce significativamente la presión arterial sistólica como indicador de disminución de la ansiedad. No se encontró diferencia significativa en términos de control de dolor y ansiedad, ni en requerimiento de sedación o analgesia adicional, pero al ser esta una técnica sencilla, económica y que ejerce efectos de relajación, puede implementarse en el ámbito hospitalario.

\section{Financiamiento}

Los autores no recibieron patrocinio para llevar a cabo este artículo.

\section{Conflicto de intereses}

Los autores declaran no tener conflicto de intereses.

\section{Responsabilidades éticas}

Protección de personas y animales. Los autores declaran que los procedimientos seguidos se conformaron a las normas éticas del comité de experimentación humana responsable y de acuerdo con la Asociación Médica Mundial y la Declaración de Helsinki.
Confidencialidad de los datos. Los autores declaran que han seguido los protocolos de su centro de trabajo sobre la publicación de datos de pacientes.

Derecho a la privacidad y consentimiento informado. Los autores han obtenido el consentimiento informado de los pacientes y/o sujetos referidos en el artículo. Este documento obra en poder del autor de correspondencia.

\section{Bibliografía}

1. Lovich-Sapola J, Smith CE, Brandt CP. Postoperative pain control. Surg Clin North Am. 2015;95:301-18.

2. Khan RS, Ahmed K, Blakeway E, Skapinakis P, Nihoyannopoulos L, Macleod K, et al. Catastrophizing: a predictive factor for postoperative pain. Am J Surg. 2011;201:122-31.

3. Dolin SJ, Cashman JN, Bland JM. Effectiveness of acute postoperative pain management: I. Evidence from published data. Br J Anaesth. 2002; 89:409-23.

4. Caumo W, Schmidt AP, Schneider CN, Bergmann J, Iwamoto CW, Adamatti LC, et al. Preoperative predictors of moderate to intense acute postoperative pain in patients undergoing abdominal surgery. Acta Anaesthesiol Scand. 2009;46:1265-71.

5. Ruales SAM, Ortega P, Sánchez A, Guéraçague R, Palomeque A, Endara $P$. El uso de ondas sonoras reduce los niveles de estrés y dolor agudo postquirúrgico. Rev Mex Anest. 2016;39:50-7.

6. Ludwin DB. Setting up an ambulatory regional anesthesia program for orthopedic surgery. Anesthesiol Clin. 2014;32:911-21.

7. Lehmann $\mathrm{M}$, Monte $\mathrm{K}$, Barach $\mathrm{P}$, Kindler $\mathrm{CH}$. Postoperative patient complaints: a prospective interview study of 12,276 patients. J Clin Anesth. 2010;22:13-21.

8. Bailey L. Strategies for decreasing patient anxiety in the perioperative setting. AORN J. 2010;92:445-60.

9. Lautenbacher S, Huber C, Schofer D, Kunz M, Parthum A, Weber PG, et al. Attentional and emotional mechanisms related to pain as predictors of chronic postoperative pain: a comparison with other psychological and physiological predictors. Pain. 2010;151:722-31.

10. Graversen $M$, Sommer T. Perioperative music may reduce pain and fatigue in patients undergoing laparoscopic cholecystectomy. Acta Anaesthesiol Scand. 2013;57:1010-6.

11. Korhan EA, Uyar M, Eyigor C, Hakverdioglu Yont G, Celik S, Khorshid L. The effects of music therapy on pain in patients with neuropathic pain. Pain Manag Nurs. 2014;15:306-14.

12. Thoma MV, La Marca R, Brönnimann R, Finkel L, Ehlert U, Nater UM. The effect of music on the human stress response. PLoS One. 2013;8:e70156.

13. Vaajoki $A$, Kankkunen $P$, Pietilä A-M, Vehviläinen-Julkunen K. Music as a nursing intervention: effects of music listening on blood pressure, heart rate, and respiratory rate in abdominal surgery patients. Nur Health Sci. 2011;13:412-8.

14. Aragon D, Farris $C$, Byers JF. The effects of harp music in vascular and thoracic surgical patients. Altern Ther Health Med. 2002;8:52-4, 6-60.

15. Leys C, Schumann S. A nonparametric method to analyze interactions: the adjusted rank transform test. J Exper Social Psychol. 2010;46: 684-8.

16. Bridgestock $C$, Rae CP. Anatomy, physiology and pharmacology of pain. Anaesthesia \& Intensive Care Medicine. 2013;14:480-3.

17. Cole LC, LoBiondo-Wood G. Music as an adjuvant therapy in control of pain and symptoms in hospitalized adults: a systematic review. Pain Manag Nurs. 2014;15:406-25.

18. Kühlmann AYR, Rooij AD, Kroese LF, Dijk MV, Hunink MGM, Jeekel J. Meta-analysis evaluating music interventions for anxiety and pain in surgery. Br J Surg. 2018;105:773-83.

19. Thoma MV, La Marca R, Bronnimann R, Finkel L, Ehlert U, Nater UM. The effect of music on the human stress response. PLoS One. 2013; 8:e70156.

20. Eckhouse DR, Hurd M, Cotter-Schaufele S, Sulo S, Sokolowski M, Barbour $L$. A randomized controlled trial to determine the effects of music and relaxation interventions on perceived anxiety in hospitalized patients receiving orthopaedic or cancer treatment. Orthop Nurs. 2014;33:342-51.

21. Ames N, Shuford R, Yang L, Moriyama B, Frey M, Wilson F, et al. Music listening among postoperative patients in the intensive care unit: a randomized controlled trial with mixed-methods analysis. Integr Med Insights. 2017;12:1178633717716455.

22. Gezginci E, lyigun E, Yalcin S, Bedir S, Ozgok IY. Comparison of two different distraction methods affecting the level of pain and anxiety during 
extracorporeal shock wave lithotripsy: a randomized controlled trial. Pain Manag Nurs. 2018;19:295-302.

23. Kwekkeboom KL. Music versus distraction for procedural pain and anxiety in patients with cancer. Oncol Nurs Forum. 2003:30:433-40.

24. Finlay KA, Wilson JA, Gaston P, AI-Dujaili EAS, Power I. Post-operative pain management through audio-analgesia: investigating musical constructs. Psychology of Music. 2015;44:493-513.

25. Nilsson U, Rawal N, Unosson M. A comparison of intra-operative or postoperative exposure to music - a controlled trial of the effects on postoperative pain. Anaesthesia. 2003;58:699-703.

26. Cheung CWC, Yee AWW, Chan PS, Saravelos SH, Chung JPW, Cheung LP, et al. The impact of music therapy on pain and stress reduction during oocyte retrieval - a randomized controlled trial. Reprod Biomed Online. 2018;37:145-52.

27. Bradt J, Dileo C, Shim M. Music interventions for preoperative anxiety. Cochrane Database Syst Rev. 2013(6):CD006908.

28. Bradt J, Dileo C, Potvin N. Music for stress and anxiety reduction in coronary heart disease patients. Cochrane Database Syst Rev. 2013; (12):CD006577.
29. Singh VP, Rao V, Prem V, Sahoo RC, Keshav Pai K. Comparison of the effectiveness of music and progressive muscle relaxation for anxiety in COPD - a randomized controlled pilot study. Chron Respir Dis. 2009;6:209-16.

30. Bradt J, Dileo C, Potvin N. Music for stress and anxiety reduction in coronary heart disease patients. Cochrane Database Syst Rev. 2013; (12):CD006577.

31. Ugras GA, Yildirim G, Yuksel S, Ozturkcu Y, Kuzdere M, Oztekin SD. The effect of different types of music on patients' preoperative anxiety: a randomized controlled trial. Complement Ther Clin Pract. 2018;31:158-63.

32. Song M, Li N, Zhang X, Shang Y, Yan L, Chu J, et al. Music for reducing the anxiety and pain of patients undergoing a biopsy: a meta-analysis. J Adv Nurs. 2018;74:1016-29.

33. Gallagher LM, Gardner V, Bates D, Mason S, Nemecek J, DiFiore JB et al. Impact of music therapy on hospitalized patients post-elective orthopaedic surgery: a randomized controlled trial. Orthop Nurs. 2018; 37:124-33.

34. Ellis RJ, Thayer JF. Music and autonomic nervous system (dys)function. Music Percept. 2010;27:317. 